\title{
Zur Kenntnis der Bohn-Sehmidt'schen Reaktion in der Benzolreihe und über die Bestimmung des Stickstoffs nach Kjeldahl in Nitroverbindungen
}

von

\author{
Dr. Alfred Eckert.
}

Aus dem chemischen Laboratorium der k. k. deutschen Universität in Prag.

(Vorgelegt in der Sitzung am 24. April 1913.)

Daß durch Erhitzen der verschiedenen Nitroanthrachinone und Nitronaphthaline mit konzentrierter Schwefelsäure Farbstoffe entstehen, ist eine längst konstatierte Tatsache. Durch die Patente der Badischen Anilin- und Sodafabrik ist bekannt geworden, daß man zu technisch wertvollen Farbstoffen kommt, wenn man auf Dinitroanthrachinon Schwefelsesquioxyd, d. h. eine Lösung von Schwefel in rauchender Schwefelsäure einwirken läßt. Über den Verlauf dieser. merkwürdigen Reaktion klärt uns eine Arbeit von R. E. Schmidt und Gattermann ${ }^{1}$ auf. Es wird darin gezeigt, daß primär Hydroxylaminoderivate entstehen, die durch die konzentrierte Schwefelsäure zu Aminooxyverbindungen umgelagert werden. Durch zahlreiche Patente der Elberfelder Farbenfabriken ist gezeigt worden, daß der Reaktionsverlauf bei der Darstellung des Naphthazarins aus Dinitronaphthalin ein analoger ist, indem man auch hier bei gemäßigter Einwirkung von Oleum und Schwefel Zwischenprodukte isolieren konnte, die den Charakter von Aminonaphtholen haben.

1 B., 29, 2934. 
Der Gedanke, diese Reaktion auf Benzolderivate zu übertragen, lag nahe und es soll in vorliegender Arbeit über die Einwirkung von Schwefelsesquioxyd auf Nitrobenzol berichtet werden.

Läßt man Schwefelsäure für sich allein bei höherer Temperatur auf Nitrobenzol einwirken, so lassen sich außer Nitrobenzolsulfosäure und unverändertem Nitrobenzol keine krystallinischen Reaktionsprodukte auffinden. $\mathrm{Zu}$ demselben Resultate gelangt man, wenn man rauchende Schwefelsäure bei den gleichen Reaktionsbedingungen auf Nitrobenzol einwirken läßt. Verwendet man aber eine Lösung von Schwefel in rauchender Schwefelsäure, so ist der Reaktionsverlauf ein analoger, wie in den oben angeführten Fällen; nur entstehen hier noch einige Körper, von denen Analoga weder in der Anthrachinon- noch in der Naphthalinreihe beobachtet wurden.

Die Aufarbeitung des Reaktionsgemisches gestaltet sich nur bei genauer Einhaltung folgender Bedingungen $\mathrm{zu}$ einer einfachen.

$20 \mathrm{~g}$ Nitrobenzol werden in $40 \mathrm{~g}$ Monohydrat unter Kühlung gelöst. Diese Lösung wird nun in kleinen Partien in eine Lösung von $6 \mathrm{~g}$ Schwefel in $200 \mathrm{~g} 35$ - bis 40prozentigem Oleum eingetragen. Da die Reaktion von starker Wärmeentwicklung begleitet ist, wird durch gute Kühlung Sorge getragen, daß die Temperatur $20^{\circ}$ nicht überschreitet. Nach dem Eintragen wird noch eine halbe bis eine Stunde stehen gelassen und dann das Ganze auf Eis gegossen. Nachdem etwas unveränderter Schwefel abfiltriert wurde, neutralisiert man die Hauptmenge der Schwefelsäure mit Baryt. Nach dem Abfiltrieren des Bariumsulfates wird die Flüssigkeit auf die Hälfte eingedampft und mit Äther extrahiert. Der Äther nimmt eine in langen Nadeln krystallisierende Substanz auf, die nach viermaligem Umkrystallisieren bei $183^{\circ}$ schmilzt. Die Substanz erwies sich als $p$-Aminophenol.

$0.2206 \mathrm{~g}$ geben $25 \cdot 2 \mathrm{~cm}^{3}$ Stickstoff bei $20^{\circ}$ und $750 \mathrm{~mm}$

$\mathrm{N}$ ber. $12 \cdot 84 \%$, gef. $12 \cdot 92 \%$.

Ein Mischungsschmelzpunkt mit reinem $p$-Aminophenol zeigte keine Depression. Außerdem gab die Substanz die 
charakteristischen Reaktionen des $p$-Aminophenols mit Lauge und mit Eisenchlorid. Es ist wohl anzunehmen, daß das Nitrobenzol primär zu Phenylhydroxylamin reduziert wird, aus dem dann durch die konzentrierte Schwefelsäure das $p$-Aminophenol entsteht, da ja auch bei der Elektrolyse von Nitrobenzol in konzentrierter schwefelsaurer Lösung $p$-Aminophenol entsteht, was ebenfalls auf eine Umlagerung des primär entstehenden Phenylhydroxylamins zurückgeflihrt wird. Die Ausbeute an $p$-Aminophenol ist eine sehr geringe. Aus $20 \mathrm{~g}$ Nitrobenzol erhält man $0.7 \mathrm{~g}$ reines Produkt.

Die ausgeätherte Lauge wird nun zur. Entfernung der letzten Reste der Schwefelsäure mit einer Bariumchloridlösung versetzt und nach Entfernung des Bariumsulfates vollständig zur Trockene eingedampft. Der schwarze amorphe Rückstand wird nun mehrmals mit Alkohol ausgekocht. Der Rückstand wird nun aus Wasser fraktioniert krystallisiert. Es gelingt so unschwer, zwei Fraktionen zu erhalten. Der leichter lösliche Teil enthält Bariumsaize von Sulfosäuren. Man löst dieselben in Wasser und versetzt mit Salzsäure. Beim Erkalten scheiden sich feine braune Nadeln aus, die nach mehrmaligem Umkrystallisieren rein weiß wurden. Diese so erhaltene Substanz wurde durch ihre Eigenschaften und durch die Analyse als $p$-Aminophenol-2-sulfosäure erkannt. Die Ausbeute an reiner Säure war $1.3 \mathrm{~g}$. Die Stickstoffbestimmung wurde nach Kjeldahl ausgeführt.

N. $0.3086 \mathrm{~g}$ verbrauchen $1 \cdot 66 \mathrm{~cm}^{3} 1 / 10$ normale Salzsäure. Ber. $7 \cdot 40 \%$, gef. $5 \cdot 730 \%$.

S. $0.2342 g$ geben $0 \cdot 3046 g \mathrm{BaSO}_{4}$, ber. $16 \cdot 93 \%$, gef. $16 \cdot 83 \% \%_{0}$.

Die Mutterlaugen von der $p$-Aminophenolsulfosäure wurden konzentriert. Dabei schied sich noch eine geringe Menge dieser in Wasser sehr schwer löslichen Säure aus. Das Filtrat davon wurde mit einer Lösung von Kaliumsulfat versetzt, vom ausgeschiedenen Bariumsulfat abfiltriert und eingedampft. Durch mehrmaliges Umkrystallisieren wurden schöne Nadeln erhalten, die als $m$-Nitrobenzolsulfosaures Kalium identifiziert werden konnten.

K. $0 \cdot 1782 g$ geben $0 \cdot 204 g \mathrm{~K}_{2} \mathrm{SO}_{4}$, ber. $16 \cdot 190 / 0 \mathrm{~K}$, gef. $16 \cdot 03 \% \mathrm{~K}$. 
Die Ausbeute war $1 \cdot 2 g$ reines Kaliumsalz.

Der oben erwähnte schwarze Rückstand der Bariumsalze wurde nun aus zirka 10 prozentiger Salzsäure fraktioniert umkrystallisiert. Nach vielen Krystallisationen wurden zwei Körper erhalten. Der eine, schwerlösliche Anteil bildet grünschillernde gekrümmte Nadeln. Sie erwiesen sich als ein Chlorhydrat. Zwecks Gewinnung der freien Base wurden sie in verdünnter Salzsäure gelöst und nun Ammoniak zugesetzt. Man darf nur so viel Ammoniak zusetzen, bis die Flüssigkeit gerade neutralisiert ist, da sich der ausfallende braunrote Niederschlag im Überschusse des Fällungsmittels wieder auf löst. Der Niederschlag bildet nach dem Trocknen ein grünschillerndes braunrotes Pulver, das in den gebräuchlichen Lösungsmitteln nur sehr schwer löslich ist. Leicht löst es sich in konzentrierter Schwefelsäure mit blauer Farbe und in Alkalien und Natriumacetat mit blauvioletter Farbe und brauner Fluoreszenz. Mit einer Suspension von Bariumkarbonat gekocht, löst es sich violett. Nach dem Abfiltrieren des überschüssigen Bariumkarbonates und Eindampfen der Laugen hinterbleibt ein Bariumsalz in braunen Krusten. Die Ausbeute an dieser Substanz ist eine sehr kleine; es wurden aus $60 \mathrm{~g}$ Nitrobenzol nur $0.25 g$ erhalten. Die Schwefelbestimmung in der freien Base wurde nach Liebig ausgeführt.

S. $0.1137 g$ geben $0 \cdot 1118 g$ BaSO, 1 , gef. $13 \cdot 50 \% / 0$ a, ber. fiir $\mathrm{C}_{12} \mathrm{H}_{11} \mathrm{NSO}_{2}$ $13 \cdot 98 \%$ Ba.

Das Bariumsalz enthielt: Ba. $\cdot 0 \cdot 0783 \mathrm{~g}$ geben $0 \cdot 064 \mathrm{~g} \mathrm{BaSO}_{4}$, ber. $35 \cdot 86 \%$, gef. $35 \cdot 37 \% \mathrm{Ba}$.

Aus den erhaltenen Analysenzahlen und den Eigenschaften dieses Körpers kán man schließen, daß es offenbar identisch ist mit dem von Bernthsen ${ }^{1}$ aus $p$-Aminophenol dargestellten Thionol. $\left.\left(\mathrm{NH} K \sum_{\mathrm{C}_{6} \mathrm{H}_{3} \mathrm{OH}}^{\mathrm{C}}\right)_{6}^{\mathrm{C}_{6} \mathrm{H}_{3} \mathrm{OH}}\right)$ Die geringe Menge des erhaltenen Produktes erlaubte eine weitere Reinigung nicht, so daß die erhaltenen Zahlen gegen die theoretischen Werte zu niedrig sind. 
Was den Mechanismus der Bildung des Thionols anbelangt, so kann man sich vorstellen, daß aus dem primär entstandenen $p$-Amidophenol zunächst das Thionolin $\mathrm{NH}\left\langle\underset{\mathrm{C}_{6} \mathrm{H}_{4} \mathrm{OH}}{\sum_{\mathrm{S}}^{\mathrm{C}_{6} \mathrm{H}_{4} \mathrm{NH}_{2}}}\right.$ entsteht, welches dann durch die konzentrierte Schwefelsäure unter Abspaltung von Ammoniak in das Thionol übergeht.

Der leichter lösliche Anteil von Thionol bildet im reinen Zustande graublaue Krystalle, die sich in Wasser nur sehr schwer mit blauer Farbe lösen. In Alkalien sind sie mit gleicher Farbe bedeutend leichter löslich. Der Körper war schwefel- und stickstoffhaltig. Die wässerige Lösung reagierte sauer und lieferte mit einer Suspension von Bariumkarbonat unter Aufbrausen ein Bariumsalz, das in Wasser sehr schwer mit roter Farbe löslich ist. Die Analyse lieferte folgende Zahlen:

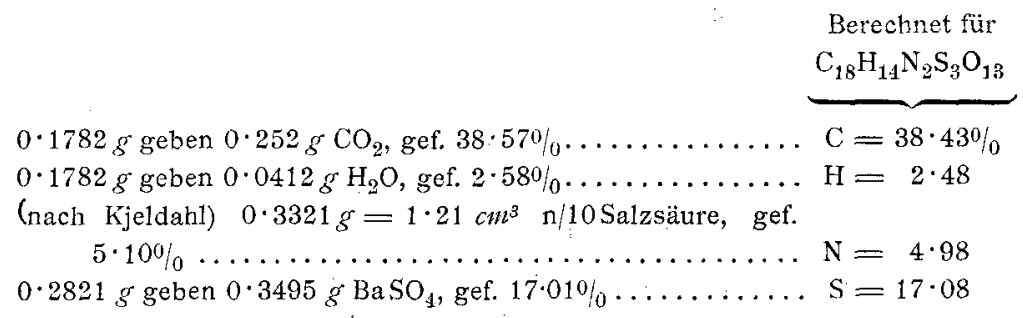

Daraus kann man folgende Bruttoformel rechnen:

$$
\mathrm{C}_{18} \mathrm{H}_{14} \mathrm{~N}_{2} \mathrm{~S}_{3} \mathrm{O}_{13}
$$

Durch Kochen mit Salzsäure und Zinnchlorür wird der Körper zerstört und beim Erkalten der Lösung krystallisiert $p$-Aminophenol-2-sulfosäure aus, während die Mutterlaugen einen Körper enthalten, der mit Eisenchlorid eine starke Rotfärbung gibt, der sich aber seiner großen Zersetzlichkeit halber nicht in reinem Zustande isolieren läßt. In seinem ganzen Verhalten zeigte diese Substanz die größte Ähnlichkeit mit einem mehrwertigen Phenol. Doch gelang es nicht, ein Acetyl-oder Benzolderivat desselben in reiner Form zu fassen. Es wurden stets nur amorphe Substanzen erhaiten, in denen sich aber der Acylrest nachweisen ließ. 
Versetzt man eine Lösung der ursprünglichen Substanz in verdünnter Salzsäure mit Jodkaliumlösung, so wird Jod in Freiheit gesetzt. Wir haben es also offenbar mit einem Chinonderivat zu tun. Es hat also hier analog wie bei der Darstellung des Naphthazarins (5-6-Dioxy-1-4-Naphthochinon) aus Dinitronaphthalin auch hier eine Oxydation zum Chinonderivate stattgefunden. Nun hat $Z$ ink ${ }^{1}$ gezeigt, daß Chinon sich mit Aminen verbindet. Bedenkt man, daß bei der geschilderten Reaktion auch $p$-Amidophenol-2-sulfosäure entsteht, so wird man wohl diesen Körper in Analogie mit den Aminderivaten des Chinons folgende Konstitution zusprechen können:

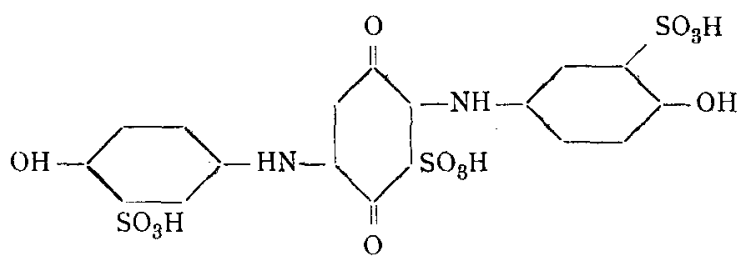

In Übereinstimmung damit steht die Tatsache, daß man bei der oben erwähnten Spaltung der Substanz mit Zinnchlorür $62 \cdot 3 \% p$-Aminophenol-2-sulfosäure erhält statt der berechneten Menge $67 \cdot 2 \%$. Bei der Titration des aus angesäuerter Jodkaliumlösung ausgeschiedenen Jods wurden stimmende Zahlen nicht erhalten. Die Substanz wird nämlich dabei zerstört, indem primär ebenfalls $p$-Aminophenol-2-sulfolsäure entsteht, auf die dann das gebildete Jod oxydierend einwirkt. Die Substanz konnte auch durch Erhitzen mit Lauge gespalten werden, doch gelang es nur $p$-Aminophenol-2-sulfosäure $z u$ fassen, während das zweite Spaltungsprodukt durch Oxydation verharzte. Die Ausbeute an dieser Substanz ist aus $20 \mathrm{~g}$ Nitrobenzol $1 \cdot 3 \mathrm{~g}$.

Es wurden nun Versuche gemacht, diesen Körper synthetisch darzustellen. Es sei jedoch gleich bemerkt, daß man bei allen Versuchen zwar Körper mit ähnlichen Eigenschaften erhält, daß man aber weder durch Sulfurieren von Dianilidochinon noch durch Vereinigung von Chinonsulfosäure mit $p$-Aminophenol-2-sulfosäure das gewünschte Produkt erhalten konnte. 
Die oben erwähnten in Alkohol löslichen Partien des Reaktionsproduktes hinterbleiben beim Abdestillieren desselben in Form von schwarzgrünen amorphen Massen, die auf keine Weise krystallisiert zu erhalten waren. Sie sind offenbar aus dem $p$-Aminophenol und der $p$-Aminophenol-2-sulfosäure durch die oxydierende Wirkung des freien Schwefeltrioxyds entstanden, sie haben in ihrem ganzen Verhalten einige Ähnlichkeit mit dem Emeraldin.

Den Verlauf der Reaktion kann man also durch folgendes Schema ausdrücken.

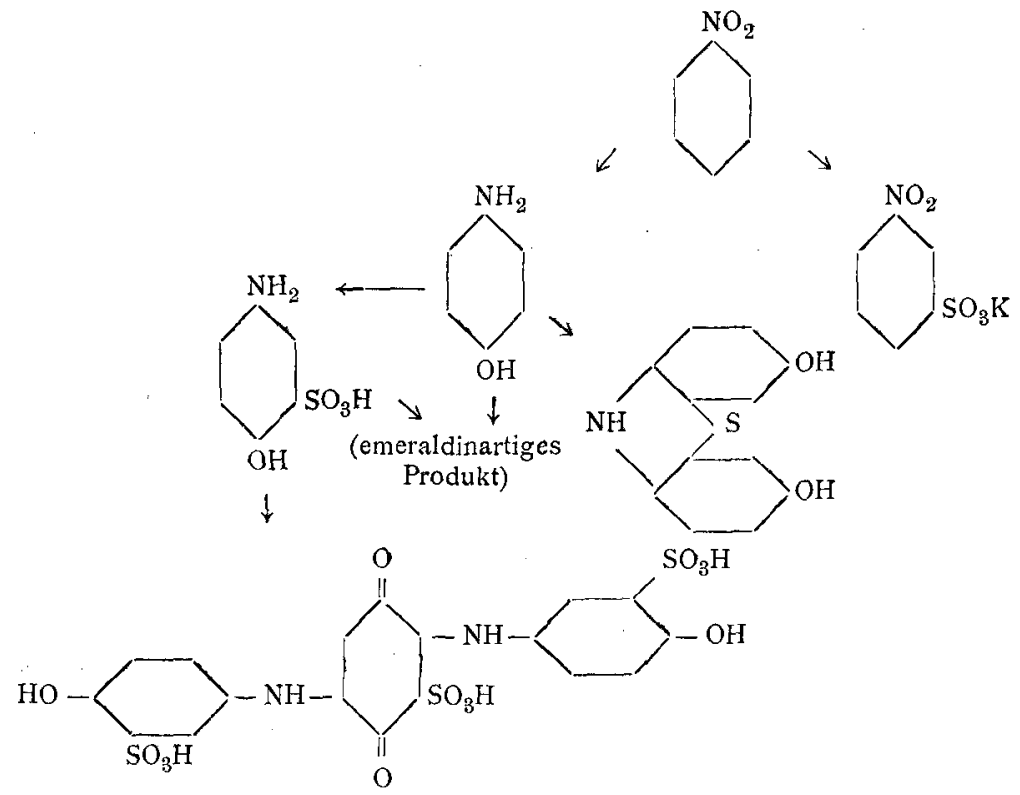

Anhang.

Da durch die Einwirkung des Schwefelsesquioxydes auf die Nitrokörper die Nitrogruppe so leicht reduziert wird, so wurde der Versuch gemacht, ob man diese Methode nicht auch zur quantitativen Bestimmung des Stickstoffs nach der Kjeldahl'schen Methode heranziehen könnte. Obwohl dieses Problem bereits als gelöst zu bezeichnen ist, haften den dafür vorgeschlagenen Methoden Übelstände an, was schon daraus hervorgeht, daß sie nur relativ selten angewendet werden. Nach 
der folgenden Methode wurden die angeführten Nitrokörper analysiert. Es wurde folgendermaßen gearbeitet:

0.2 bis $0.5 \mathrm{~g}$ der Substanz werden mit $0.4 \mathrm{~g}$ Schwefel in einem Kjeldahl-Kolben durch Umschwenken gemischt und dann 15 bis $20 \mathrm{~cm}^{3}$. 30 bis $40 \%$ Oleum zugesetzt. Man erwärmt nun eine Stunde auf dem siedenden Wasserbade. Nach dieser Zeit ist die Reduktion beendet und es wird nun in der gewöhnlichen Weise weiter gearbeitet.

Nitrobenzol. $0 \cdot 212 \mathrm{~g}$. Zur Neutralisation des gebildeten Ammoniak wurden $1 \cdot 75 \mathrm{~cm}^{3} 1 / 10$ normale Salzsäure gebraucht.

Ber. $11 \cdot 380 \% \mathrm{~N}$, gef. $11 \cdot 56 \% \mathrm{~N}$.

$p$-Bromnitrobenzo $1.0 .3212 \mathrm{~g}$ verbrauchen $1.55 \mathrm{~cm}^{3} 1 / 10$ normale Salzsäure. Ber. $6 \cdot 93 \%$ N, gef. $6 \cdot 75 \%$ N.

1, 4-Dichlor-2-Nitrobenzo1. $0.3122 \mathrm{~g}$ verbrauchen $1.65 \mathrm{~cm}^{3} \quad 1 / 10$ normale

Salzsäure.

Ber. $7 \cdot 290 / 0 \mathrm{~N}$, gef. $7 \cdot 170 / 0 \mathrm{~N}$.

$o$-Nitropheno1, $0 \cdot 4825 \mathrm{~g}$ verbrauchen $3 \cdot 5 \mathrm{~cm}^{3} 1 / 10$ normale Salzsäure.

Ber. $10 \cdot 070 / 0 \mathrm{~N}$, gef, $10 \cdot 350 \% \mathrm{~N}$.

Pikrinsäure. $0 \cdot 2433 \mathrm{~g}$ verbrauchen $3 \cdot 2 \mathrm{~cm}^{3} 1 / 10$ normale Salzsäure.

Ber. $18 \cdot 340 \%_{0} \mathrm{~N}$, gef. $18 \cdot 44 \% \%_{0} \mathrm{~N}$.

1, 3-Dinitrobenzo1. $0 \cdot 1691 \mathrm{~g}$ verbrauchen $2 \cdot 05 \mathrm{~cm}^{3} 1 / 10$ normale Salzsäure.

Ber. $16 \cdot 71 \%$ N, gef. $16 \cdot 90 \%$ N.

1, 3-Nitranilin. $0 \cdot 2998 \mathrm{~g}$ verbrauchen $4 \cdot 4 \mathrm{~cm}^{3} 1 / 10$ normale Salzsäure.

Ber. $20 \cdot 43 \%$ N, gef. $20 \cdot 560 \% \mathrm{~N}$.

Nitrosodimethylanilin. $0 \cdot 281 \mathrm{~g}$ verbrauchen $3 \cdot 65 \mathrm{~cm}^{3} 1 / 10$ normale Salzsäure.

Ber. $20 \cdot 43 \% \%_{0} \mathrm{~N}$, gef. $20 \cdot 560 \%_{0} \mathrm{~N}$.

1-Nitronaphthalin. $0 \cdot 2709 \mathrm{~g}$ verbrauchen $1.58 \mathrm{~cm}^{3} 1 / 10$ normale Salzsäure. Ber. $8 \cdot 090 / 0 \mathrm{~N}$, gef. $8 \cdot 16 \%$ N.

1,5-Dinitronaphtha1 in. $0 \cdot 3148 \mathrm{~g}$ verbrauchen $2 \cdot 91 \mathrm{~cm}^{31} / 10^{\text {normale Salzsäure. }}$ Ber. $12 \cdot 84 \%$ N, gef. $12 \cdot 930 \% \mathrm{~N}$.

1-Nitroanthrachin on. $0 \cdot 4326 g$ verbrauchen $1.78 \mathrm{~cm}^{3} 1 / 10$ normale Salzsäure. Ber. $5 \cdot 53 \%$ N, gef. $5 \cdot 76 \%$ N

1, 5-Dinitroanthrachinon. $0.4252 \mathrm{~g}$ verbrauchen $2 \cdot 91 \mathrm{~cm}^{3} 1 / 10$ normale Salzsäure.

Ber. $9 \cdot 39 \%$ N, gef. $9 \cdot 570 \%_{0} \mathrm{~N}$.

Es wurde auch versucht, diese Methode auf aliphatische Nitroverbindungen und auf anorganische Nitrate anzuwenden. Aber in beiden Fällen wurden viel zu niedrige Zahlen erhalten, so daß auf die geschilderte Weise nur aromatische Nitroverbindungen analysiert werden können. 